\title{
Gating mechanism of human N-type voltage-gated calcium channel
}

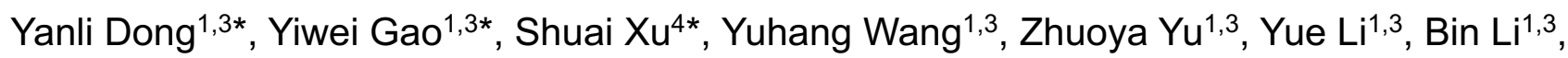
Bei Yang ${ }^{1,3}$, Xuejun Cai Zhang ${ }^{1,3}$, Daohua Jiang ${ }^{5 * *}$, Zhuo Huang ${ }^{4,6 * *}$, Yan Zhao ${ }^{1,2,3 * *}$

${ }^{1}$ National Laboratory of Biomacromolecules, CAS Center for Excellence in

Biomacromolecules, Institute of Biophysics, Chinese Academy of Sciences, Beijing 100101, China

2 State Key Laboratory of Brain and Cognitive Science, Institute of Biophysics, Chinese Academy of Sciences, 15 Datun Road, Beijing, 100101, China

${ }^{3}$ College of Life Sciences, University of Chinese Academy of Sciences, Beijing 100049, China

${ }^{4}$ State Key Laboratory of Natural and Biomimetic Drugs, Department of Molecular and Cellular Pharmacology, School of Pharmaceutical Sciences, Peking University Health Science Center, Beijing, 100191, China

${ }^{5}$ Laboratory of Soft Matter Physics, Institute of Physics, Chinese Academy of Sciences, Beijing 100190, China

${ }^{6}$ IDG/McGovern Institute for Brain Research, Peking University, Beijing, 100871, China

* These authors contribute equally to this project.

** Correspondence emails: zhaoy@ibp.ac.cn (Y.Z.), huangz@hsc.pku.edu.cn (Z.H.), jiangdh@iphy.ac.cn (D.J.) 


\section{Abstract}

$\mathrm{N}$-type voltage-gated calcium ( $\mathrm{Cav}$ ) channels mediate $\mathrm{Ca}^{2+}$ influx at the presynaptic terminals in response to action potential and play vital roles in synaptogenesis, neurotransmitter releasing, and nociceptive transmission. Here we elucidate a cryo-electron microscopy (cryo-EM) structure of the human Cav2.2 complex at resolution of $2.8 \AA$. This complex structure reveals how the Cav2.2, $\beta 1$, and $\alpha 2 \delta 1$ subunits are assembled. In our structure, the second voltage-sensing domain (VSD) is stabilized at a resting-state conformation, which is distinct from the other three VSDs of Cav2.2 as well as activated VSDs observed in previous structures of Cav channels. The structure also shows that the intracellular gate formed by S6 helices is closed, and a W-helix from the DII-III linker is determined to act as a blocking-ball that causes closed-state inactivation in Cav2.2. Collectively, our structure provides previously unseen structural insights into fundamental gating mechanisms of Cav channels. 


\section{Introduction}

Voltage-gated calcium channels (Cav channels) are a type of essential mediators to convert action potential into influx of $\mathrm{Ca}^{2+}$ ions, a crucial secondary messenger to regulate a variety types of cellular events, such as muscle contraction, secretion of the neurotransmitters, cell division, differentiation, and apoptosis ${ }^{1-4}$. Cav channels are generally categorized into two groups according to their activation threshold, i.e., highvoltage activated (HVA) and low-voltage activated (LVA) Cav channels. Based on their sequence homology, Cav channels in mammals contain ten members, which are further classified into three subfamilies (Cav1, Cav2, and Cav3) and six types (L-, P/Q-, N-, R-, Ttypes) ${ }^{5-7}$. The N-type Cav channel, also called Cav2.2, is a HVA channel, belongs to the Cav2 subfamily (containing P/Q-, N-, and R-type), and is exclusively expressed in the central and peripheral neurons ${ }^{8}$. The Cav2.2 is predominantly located in the pre-synaptic terminals and mediates the $\mathrm{Ca}^{2+}$ influx that triggers neurotransmitter release at the fast synapses ${ }^{9,10}$. Dysfunctions of Cav2.2 channels alter neuronal functions and lead to diseases, such as myoclonus-dystonia-like syndrome ${ }^{11}$. Moreover, the Cav2.2 channel plays a critical role in spinal nociceptive signaling, and thus it has become an important drug target for chronic pain treatment.

Molecular mechanisms of the Cav channels have been studied extensively for decades, including recent structural studies of L-type Cav1.1 isolated from rabbit skeletal muscle ${ }^{12-15}$ and human T-type Cav3.1 ${ }^{16}$. These studies revealed structural features of the Cav channels at their inactivated state with all the voltage sensing domains (VSD।-VSDIV) adopting an activated 'up' conformation. The structures of the Cav1.1 complex also elucidate the assembly of Cav channel with auxiliary $\beta$ and $\alpha 2 \delta$ subunits. A range of ligands were determined in complexes with Cav1.1 or Cav3.1, and these structural studies provide molecular bases for ligand recognition and facilitate further rational drug development targeting Cav channels. Despite these advances in structural studies on the Cav channels, more structural insight is desirable to fully understand the molecular mechanism of the Cav2.2 channel from structural untouched Cav2 subfamily, because not only it shares low sequence identity with the available structures from the other two subfamilies, but also 
some fundamental mechanistic questions remain to be addressed. For example, whereas the available structures of Cav channels are featured with activated VSDs ${ }^{12-14,16}$, these VSDs at their resting state appear to be required to fully understand the channel gating mechanism. Moreover, the Cav2.2 harbors a distinct closed-state inactivation during repolarization ${ }^{17-19}$ and is modulated by phosphatidylinositol 4,5-bisphosphate (PIP 2$)$ molecules $20-25$. These underlying molecular mechanisms need further investigation to elucidate.

Here, we purified the recombinant human Cav2.2 (also called $\alpha$-subunit) in complex with the $\beta 1$ and $\alpha 2 \delta 1$ subunits, and determined their complex structure using single particle cryo-EM method. This structure reveals the first view of asymmetric activation of the four VSDs with the VSD॥l at its resting state, presumably locked by a $\mathrm{PIP}_{2}$-like lipid molecule. Furthermore, we identify a unique $\alpha$-helix that blocks the intracellular gate of Cav2.2, contributing to the close-state inactivation of the Cav2.2. These results enable us to gain significant novel insights into the activation and inactivation mechanisms of human Cav channels.

\section{Results and discussion}

\section{Structure determination and architecture of the Cav2.2 complex}

To investigate the architecture of the N-type Cav2.2 complex, we co-expressed human $\mathrm{N}$-terminal truncated Cav2.2, full-length wild type (WT) $\alpha 2 \delta 1$, and full-length WT $\beta 1$ in HEK 293 cells. To monitor the expression of the complex during purification, the Cav2.2 was fused with a C-terminal GFP-Twinstrep tag. In order to enhance the expression level, the intrinsically disordered N-terminal region (residues 1-64) was truncated, and we denote this construct as Cav2.2 $2^{\mathrm{EM}}$. We carried out whole-cell patch clamp experiments in HEK 293T cells to characterize the channel properties of both human WT full-length Cav2.2 and Cav2.2 $2^{\mathrm{EM}}$ constructs, in the presence of auxiliary $\beta 1$ and $\alpha 2 \delta 1$ subunits. The Cav2.2 $2^{\mathrm{EM}}$ complex shows undistinguishable gating properties to the full-length Cav2.2, in terms of voltage-dependent activation and steady-state inactivation (Fig. 1a-1b; Extended Data Fig. 1a-1b). Thus, the Cav2.2 $\mathrm{EM}$ construct was subjected to further structural and functional 
studies. The purified Cav2.2 ${ }^{\mathrm{EM}}-\alpha 2 \delta 1-\beta 1$ complex (Cav2.2 complex) displaying monodisperse peak on both SEC and SDS-PAGE further confirmed that all three subunits were present in the complex sample (Extended Data Fig. 1c-1d).

To elucidate its architecture, we carried out cryo-EM study of the Cav2.2 complex and obtained the final reconstruction at $2.8 \AA$ resolution for the Cav2.2 complex (Fig. 1c, Extended Data Fig. 2 and Extended Data Table 1). Cryo-EM map of the Cav2.2 is rich of structural features, including densities for side chains, N-glycans, disulfide bonds, and associated lipid molecules, which enabled us to unambiguously de novo build atomic models of the Cav2.2 complex (Fig. 1d and Extended Data Fig. 2e). Of approximately $118 \AA$ $\times 113 \AA \times 169 \AA$ in sizes, the structure of the Cav2.2 complex contains $\alpha, \alpha 2 \delta 1$, and $\beta 1$ subunits, and closely resembles the classic shape of Cav1.1.

The $\alpha$-subunit is composed of four transmembrane domains, $D_{1}-D_{I V}$, which form an ion conducting pore in a domain swapped fashion. Each domain is composed of six helices (S1-S6), of which S1-S4 helices form the voltage sensing domain (VSD). Four VSDs (VSDI-VSDIV) encircle the central pore formed by S5 and S6 helices from all four domains. The $\beta 1$ subunit comprises a Src homology $3(\mathrm{SH} 3)$ domain and a guanylate kinase (GK) domain. The GK domain, through its alpha-interacting domain (AID) helix, interacts with the Cav2.2 (i.e., the $\alpha$-subunit). Nevertheless, the GK domain was not well resolved in our cryoEM map, presumably due to conformational heterogeneity (Fig. $1 \mathrm{c}-1 \mathrm{~d}$ ). The $\alpha 2 \delta 1$ subunit is divided into $\alpha 2$ and $\delta$ subunits, which are linked by a disulfide bond (C404-C1059) and associate with the $\alpha$-subunit by interacting with extracellular loops (ECLs, in particular the loops between S5 and S6 helices) ECL, ECL II, ECLIII, and the S1-S2 loop from VSD. The structure of human $\alpha 2 \delta 1$ in our complex is nearly identical with rabbit $\alpha 2 \delta 1$ resolved in the Cav1.1 complex, with root-mean-square-deviation (r.m.s.d.) of $\sim 1.1 \AA$.

\section{Ion conduction pore of the Cav2.2 complex}

The central pore is made up of helices S5 and S6 from all four domains of the $\alpha$-subunit (Fig. 2a-2b). In each domain, a re-entrant P-loop is located between the S5 and S6 helices and contains two short helices, P1 and P2, connected by a short linker. Four P-loops form a funnel-like shape and line the outer entry to the pore. Four highly conserved glutamate 
residues, $E^{314}, E^{663}, E^{1365}$, and $E^{1655}$, form a selectivity-filter ring at the bottom of the funnel, which is one of the narrowest segments of the central pore (Fig. 2c). These four acidic residues, together with surrounding negatively charged residues, create a strong negative electric field strength to attract cations and repel anions. This pore region also functions as a selectivity filter to discriminate $\mathrm{Ca}^{2+}$ from other cation ions. We did identify a strong density within the selectivity filter and close to the glutamate cluster (Fig. 2d-2e).

Presumably, this piece of density represents a calcium ion, consistent with observations in previous structures of Cav channels ${ }^{12-16}$. Thus, on the extracellular side, the S5, S6 helices as well as the P-loops contribute to the formation and stability of the selectivity filter.

On the intracellular side, the pore-lining S6 helices comprise an intracellular gate to control ion permeation (Fig. 2f). The Cav2.2 complex was determined in the absence of membrane potential, and thus is likely to represent a depolarization state. The cytoplasmic ends of the S6 helices are converged and form a hydrophobic seal through a cluster of hydrophobic residues (Fig. 2f). In each of the four domains, the S5 helix is positioned proximal to the S6 helix and connects to S4 of VSD through an amphipathic horizontal helix (termed S4-S5 helix, approx. 16 residues). This S4-S5 helix interacts with the S6 helix, and thus couples S4 movement in responding to depolarization of membrane potential with gate opening.

The central pore of the Cav2.2 channel is further compared with those from the Cav1.1 (PDB ID: 5GJW), and Cav3.1 (PDB ID: 6KZP) channels. The S5-S6 ECLs exhibit nontrivial conformational discrepancy among these structures (Extended Data Fig. 3a, 3b, 3d and 3e). The Cav2.2 $\alpha$-subunit possesses shorter $E C L_{l}, E C L_{\|}$, and $E C L_{\| I}$, but this structural difference does not hamper binding of the $\alpha 2 \delta 1$ subunit. The S1-S2 loops of VSD II, also involved in interactions with the $\alpha 2 \delta 1$ subunit, are nearly identical in both Cav2.2 and Cav1.1. Therefore, the $\alpha 2 \delta 1$ subunits in both Cav2.2 and Cav1.1 complexes display similar binding geometry. In contrast, the ECLs of the Cav3.1 channel exhibit remarkable structural discrepancy from the corresponding loops of Cav1.1 and Cav2.2, thus giving rise to incompatibility of the $\alpha 2 \delta 1$ subunit with Cav3.1 (Extended Data Fig. 3b and 3e). Despite of these structural differences occurring at ECLs, the transmembrane helices S5, S6 as well as the selectivity filter of Cav2.2 are superimposable with Cav1.1 and Cav3.1, yielding 
r.m.s.d of $\sim 1.8 \AA$ for $\mathrm{Cav1.1}$ (for $332 \mathrm{C}_{\alpha}$ atom-pairs), and $\sim 1.6 \AA$ for Cav3.1 (for $300 \mathrm{C}_{\alpha}$ atom-pairs), respectively (Extended Data Fig. 3c and 3f). In particular, the intracellular gate formed by the four helix-bundle of S6 helices is nearly identical in all three structures, assuming a closed form (Extended Data Fig. 3g). The S6॥ helix of the Cav2.2 extends into the cytoplasmic, and is longer than that of the Cav1.1. Consequently, C-terminus of the S6॥ helix forms a close contact with the $\beta 1$ subunit in the Cav2.2 complex, which is not observed in the structure of Cav1.1. Furthermore, the cytoplasmic part of the $\mathrm{S} 6$ helix bends by $13^{\circ}$ compared with that in Cav1.1 (Extended Data Fig. 3h), which may affect gate opening and represent a potential regulatory mechanism of the $\beta$ subunit.

\section{Voltage sensor of the N-type Cav channel}

A hallmark feature of the Cav channel is to open or close the channel in respond to variation of the membrane potential. Surrounding the central pore, the four VSDs play essential roles in converting the electrostatic signal into conformational change of the intracellular gate. Each VSD is composed of S1-S4 transmembrane helices. Each S4 helix contains several positively charged residues, either arginine or lysine, spaced at intervals of three. Cav2.2 is a depolarization-activated channel. Upon depolarization of the membrane potential, the gating charges on the S4 helix move toward the extracellular cavity of the VSD. The two cavities are separated by a highly conserved hydrophobic constriction site. When the gating charges pass through this hydrophobic seal, existing interactions of the gating charges with hydrophilic and negatively charged residues on surrounding helices are disrupted on one side, and new interactions are formed on the other side. The displacement of the S4 helices across the electrostatic field of the membrane potential would induce lateral movement of the S4-S5 linkers, resulting in disengagement between S5 and S6 helices and thus the channel opening.

In our structure, the S4 helices adopt 310 -helix conformation, which is consistent with observations from previous structures of voltage-gated ion channel, including $\mathrm{Ca}_{\mathrm{v}}$ and $\mathrm{Na}$ channels, allowing the side chains of gating-charge residues to be aligned on the same side of the helix surface. Using the central pore as a reference, superimposition of the structure of Cav2.2 onto that of Cav1.1 shows fairly superimposable VSD,$V_{S D_{I I I}}$ and VSDIV, 
demonstrating that they are stabilized in the same, presumably activated state (Extended Data Fig. 4a-4e). In contrast, VSD II $_{\text {of }}$ Cav2.2 exhibits discernable conformational rearrangement compared with that from the Cav1.1 structure. Taking a closer look at the four VSDs, we found that four, four, and three gating charges are located at the extracellular aqueous cavities of $\mathrm{VSD}_{\mathrm{I}}, \mathrm{VSD}_{\mathrm{III}}$, and $\mathrm{VSD}_{\mathrm{IV}}$, respectively, and interact with polar residues residing on nearby S1-S3 helices, consistent with observations from the available structures of Cav channels ${ }^{12-14,16}$ (Figs. 3a, 3c, 3d; Extended Data Figs. 4c-4e ). Strikingly, in VSD॥ only one arginine (R578) on the S4 helix is located on the extracellular side of the hydrophobic seal, but the other four conserved gating charges (R581, R584, K587, and K590) are located in the intracellular cavity, indicating that $V_{S D}$ is stabilized at the resting state. Among the basic residues, K590 is located at a position where S4 becomes unwound, and is completely exposed to the solvent (Fig. 3b). The asymmetric activation of Cav2.2 VSDs suggests that individual VSD may sense membrane potential asynchronously, which is reported to be important for eukaryotic Navactivation and inactivation ${ }^{26}$.

To investigate conformational change of the VSD॥ of Cav2.2 upon depolarization, we overlaid its structure at the resting state $\left(V_{S D}{ }_{\|}{ }^{R}\right)$ onto that of Cav1.1 at the activated state $\left(\mathrm{VSD}_{\|}{ }^{\mathrm{A}}\right)$, and found that these $\mathrm{VSD}_{\|}$structures are highly superimposable in helices $\mathrm{S} 1, \mathrm{~S} 2$, and S3 (Fig. 3e-3f). In contrast, the S4 helix slides to the intracellular side in the resting state. Consequently, extracellular halves of S3 helix bends $\sim 15^{\circ}$ toward S4 in the resting state (Fig. 3e). Using the S5 and S6 helices as a superimposition reference, the VSD॥ undergoes substantial conformational change relative to the central pore between these two states. In particular, in transition from activated state to the resting state, the cytoplasmic sides of both S1 and S2 helices in Cav2.2 rotate toward the central pore (Fig. $3 g$ ). Consequently, the intracellular and extracellular terminus of the S3 helices are remarkably displaced approaching to the central pore by $4 \AA$ and $9 \AA$, respectively. In addition to inwardly sliding, the S4 helices also slightly shift toward the pore domain (Fig. 3h). Interestingly, even though distinguishable conformational change occurs in VSD $\mathrm{II}_{\text {, the }}$ extracellular end of the S1-S2 helix hairpin forms similar interactions with helices S5 and P1 from domain III. It appears that this interaction is stabilized by a cholesterol derivative cholesteryl hemisuccinate (CHS), which is determined in both our structure of Cav2.2 and 
the structure of Cav3.1 (Extended Data Fig. 4f), consistent with earlier reports suggesting that cholesterol is important to regulating activity of Cav channels ${ }^{27,28}$.

Compared with S4 in the activated VSD ॥ from Cav1.1, the S4 helix in the resting state undergoes a sliding movement by two helical-turns, $\sim 13 \AA$, towards intracellular side (Fig. 3h). The displacement of S4 is comparable with previous observations in structures of TPC1 channel and NavAb channel (Extended Data Fig. 4h-4i) ${ }^{29-31}$. Except R5 (K590) of Cav2.2, all gating charges are facing to one side, and no rotation about the helix axis is observed, which support a sliding-helix model of voltage-dependent gating ${ }^{31,32}$. As a consequence of the S4 inwardly sliding, the S4-S5 linker tilted by $11^{\circ}$ degree towards intracellular side, and this movement generates more contacts between the S4-S5 linker and S6 helix, and thus stabilize the inner gate at its closed state.

An extra density was identified laying above the S4-S5 linker of domain II in the cryoEM map, which presumably represents a lipid with two hydrophobic tails and a 'palm'-like head group. Its head group is located at the intracellular cavity of the VSD॥ and flanked by S3 and S4 helices (Fig. 3i). Its hydrophobic tails project toward the extracellular side of the membrane (Extended Data Fig. 5a). This lipid appears to act as a plug and would block the S4 helix from upwardly sliding, reminiscent of the $\mathrm{PIP}_{2}$ that attenuates opening of the $\mathrm{K}_{v} 1.2$ channel by interacting with the S4-S5 linker ${ }^{33}$. We attempted to fit a $\mathrm{PIP}_{2}$ into the density. Whereas its hydrophobic tails and inositol ring agree well with the density, the two phosphate groups attached to the inositol ring could not be well resolved (Extended Data Fig. 5a). Considering the $\mathrm{PIP}_{2}$ is predominant in the inner-leaflet of the plasma membrane and reported to left shift the activation curve of Cav2.2, which are consistent with our structural observations, we postulate that this lipid molecule is a $\mathrm{PIP}_{2}$. However, $\mathrm{PIP}_{2}$ molecule was not robustly detected using lipid blot and mass spectrometry methods due to poor yield of the Cav2.2 complex. In a closer look at the $\mathrm{PIP}_{2}$-like lipid binding site, several positively charged residues from helices S0, S4, and S4-S5 linker provide a highly positively charged environment for $\mathrm{PIP}_{2}$ binding (Extended Data Fig. 5b-5c). Similar local environment is not observed in the other three VSDs (Extended Data Fig. 5b), implying a mechanism by

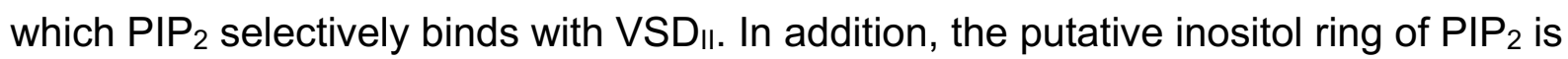
positioned proximal to the AID helix of the $\beta 1$ subunit, and this observation is consistent with 
previous investigations indicating that regulation role of $\mathrm{PIP}_{2}$ on the $\mathrm{Cav}$ channel depends on types of $\beta$ subunits ${ }^{34}$.

\section{Inactivation mechanism of the Cav2 channels}

The N-type Cav channel bears a preferential voltage-dependent closed-state inactivation $(\mathrm{CSI})^{17,18}$, featuring $\mathrm{Ca}^{2+}$ insensitive $\mathrm{U}$-shaped inactivation curve, substantial inactivation accumulated during interpulse in a two-pulse protocol and cumulative inactivation during voltage-clamped action potential trains ${ }^{17}$. In our structure, the intracellular gate is comparable with that in structures of the Cav1.1 and Cav3.1, and appears to be stabilized at a closed state (Extended Data Fig. 3g). Strikingly, an additional $\alpha$-helix was unexpectedly determined underneath the intracellular gate and flanked by the four S6-helix bundle, forming a $36^{\circ}$ angle to the membrane plane (Fig. 4a), and has not been observed in previous structures of Cav channels. The high resolution cryo-EM map aided us to register the amino-acid sequence to the model. This helix is composed of residues range from $\mathrm{A} 764$ to $\mathrm{S783}$, which belongs to the DII-III linker (Fig. 4b). The side chain of W768 points upward into the intracellular gate, forms extensive hydrophobic interactions with residues from S6 helices (Fig. 4b-4c), and thus stabilizes the intracellular gate at its closed state. Thus, we term this 6-turn helix as W-helix. In addition to the tryptophan as a plug inserting into and blocking the intracellular gate, the other residues from the W-helix extensively interact with the S6 helices, including hydrogen-bond and electrostatic interaction, to further strengthen the association of the W-helix with the intracellular gate (Fig. 4b-4c). We speculate that the freshly uncovered W-helix functions as the channel-blocking ball of the "ball-and-chain" model and is important for the stability of the intracellular gate in the closed state, thus contributing to the close-state inactivation of the Cav2.2.

To investigate the functional role of the W-helix and to validate our hypothesis, we designed two mutants, including deletion of the W-helix (Cav2.2 ${ }^{\Delta \mathrm{W} \text {-Helix}}$ ) and a point-mutation variant $\mathrm{W} 768 \mathrm{Q}\left(\mathrm{Cav} 2.2^{\mathrm{W} 768 \mathrm{Q}}\right)$, and performed whole-cell patch clamp recording experiment, with $\mathrm{Ba}^{2+}$ as charge carriers. The voltage activation curves of these two mutants were indistinguishable with the WT Cav2.2 complex, suggesting the W-helix might not participate 
in channel activation. In sharp contrast, the steady-state inactivation curves of these two variants exhibit a depolarizing shift of $\sim 24 \mathrm{mV}$ (Fig. 4d and Extended Data Fig. 6a-6b), indicating the W-helix and W768 are critical for the CSI. To investigate recovery properties of the CSI, the membrane potential of pre-pulse was held at $-40 \mathrm{mV}$, where the channel has not been opened yet. It turns out the recovery of the Cav2.2 $2^{\Delta W-H e l i x}$ variant is significantly faster than that of WT complex (Fig. 4e and Extended Data Fig. 6c), which further support that the W-helix plays vital roles in CSI of Cav2.2 channel. These results are also in line with a previous functional characterization showing that domain II-III linker is crucial for CSI of Cav2.2 ${ }^{19,35}$. To further validate our finding in a more physiologically relevant condition, we repetitively activated the Cav2.2 channels with action potential (AP) trains which were recorded in hippocampal CA1 neurons in whole-cell current-clamp configuration. As observed in previous reports ${ }^{17,19}$, we found that WT Cav2.2 channels exhibited cumulative inactivation in response to AP trains (Fig. 4f and Extended Data Fig. 6d). Interestingly, Cav2.2 ${ }^{\Delta \mathrm{W}-\text { Helix }}$ variant inactivated more slowly during the AP train, suggesting that W-helixregulated close-state inactivation contributed to Cav2.2 channel inactivation during the AP trains, thus may play an important role in short-term synaptic plasticity ${ }^{36}$. Collectively, Whelix is manifested to be essential for CSI of the Cav2.2 channel. Interestingly, the W-helix is only conserved in the Cav2 subfamily (Extended Data Fig. 7), suggesting that P/Q-type and R-type Cav channels may adopt the same inactivation mechanism.

\section{Method}

\section{Whole-cell Voltage-clamp recordings of Cav2.2 channels in HEK 293T Cells}

HEK 293T cells were cultured with Dulbecco's Modified Eagle Medium (DMEM) (Gibco) added $15 \%(\mathrm{v} / \mathrm{v})$ fetal bovine serum (FBS) (PAN-Biotech) at $37^{\circ} \mathrm{C}$ with $5 \% \mathrm{CO}$. The cells were grown in the culture dishes $(\mathrm{d}=3.5 \mathrm{~cm})$ (Thermo Fisher Scientific) for $24 \mathrm{~h}$ and then transiently transfected with $2 \mu \mathrm{g}$ control or mutant plasmids containing human $\mathrm{N}$-type calcium channel isoform $\alpha 1 \mathrm{~B}, \beta 1, \alpha 2 \delta 1$ and GFP using $1.5 \mu \mathrm{g}$ Lipofectamine 2000 Reagent (Thermo Fisher Scientific). Experiments were performed 12 to 24 hours post transfection at room temperature $\left(21 \sim 25^{\circ} \mathrm{C}\right)$ as described previously ${ }^{37}$. In brief, cells were placed on a 
glass chamber containing $105 \mathrm{mM} \mathrm{NaCl}, 10 \mathrm{mM} \mathrm{BaCl}$, $10 \mathrm{mM}$ HEPES, $10 \mathrm{mM}$ D-Glucose, $30 \mathrm{mM}$ TEA-Cl, $1 \mathrm{mM} \mathrm{MgCl}, 5 \mathrm{mM} \mathrm{CsCl},(\mathrm{pH}=7.3$ with $\mathrm{NaOH}$ and osmolarity of $\sim 310$ mosmol ${ }^{-1}$ ). Whole-cell voltage-clamp recordings were made from isolated, GFP-positive cells using $1.5 \sim 2.5 \mathrm{M} \Omega$ fire polished pipettes (Sutter Instrument) when filled with standard internal solution, containing 135 mM K-Gluconate, 10 mM HEPES, 5 mM EGTA, 2 mM $\mathrm{MgCl}_{2}, 5 \mathrm{mM} \mathrm{NaCl}, 4 \mathrm{mM} \mathrm{Mg-ATP},\left(\mathrm{pH}=7.2\right.$ with CsOH and osmolarity of $\sim 295$ mosmol$\left.^{-1}\right)$. Whole-cell currents were recorded using an EPC-10 amplifier (HEKA Electronic) at $20 \mathrm{kHz}$ sample rate and was low pass filtered at $5 \mathrm{kHz}$. The series resistance was $2 \sim 4.5 \mathrm{M} \Omega$ and was compensated $80 \sim 90 \%$. The data was acquired by PatchMaster program (HEKA Electronic).

To characterize the activation properties of Cav2.2 channels, cells were held at -100 $\mathrm{mV}$ and then a series of $200 \mathrm{~ms}$ voltage steps from $-60 \mathrm{mV}$ to $+50 \mathrm{mV}$ in $5 \mathrm{mV}$ increments were applied. The inactivation properties of Cav2.2 channels were assessed with a 10s holding-voltages ranging from $-100 \mathrm{mV}$ to $-15 \mathrm{mV}(5 \mathrm{mV}$ increments) followed by a $135 \mathrm{~ms}$ test pulse at $+10 \mathrm{mV}$. To assess the time-dependent recovery from close-state inactivation, cells were depolarized to $-40 \mathrm{mV}$ (pre-pulse) for $200 \mathrm{~ms}$ to inactivate the Cav2.2 channels, and a recovery hyperpolarization steps to $-100 \mathrm{mV}$ were applied for the indicated period (4 $\mathrm{ms} \sim 512 \mathrm{~ms}$ ), followed by a $35 \mathrm{~ms}$ test pulse at $+10 \mathrm{mV}$. To analysis the persistent inactivation of Cav2.2 channels following action potential trains, the cells were held at -100 $\mathrm{mV}$ and then a physiologically relevant AP train was applied. The AP train used to repetitively activate Cav2.2 channels was obtained from a hippocampal CA1 pyramidal neuron in whole-cell current-clamp mode ${ }^{38}$. The spike pattern contains 13 action potentials in 2 seconds (mean frequency $6.5 \mathrm{~Hz}$ ). The percentage inactivation of Cav2.2 channels was calculated from the first spike eliciting maximal current to the other spikes in the AP trains.

All data reported as mean \pm SEM. Data analyses were performed using Origin 2019b (Origin Lab Corporation), Excel 2016 (Microsoft), GraphPad Prism 6 (GraphPad Software, Inc.) and Adobe illustrator 2018 (Adobe Systems Incorporated). Steady-state activation curves were generated using a Boltzmann equation.

$$
\frac{\mathrm{g}}{\mathrm{g}_{\max }}=\frac{1}{1+\exp \left(\mathrm{V}-\mathrm{V}_{0.5}\right) / \mathrm{k}}
$$


Where $\mathrm{g}$ is the conductance, $\mathrm{g}_{\max }$ is the maximal conductance of Cav2.2 during test pulse, $\mathrm{V}$ is the test potential, $\mathrm{V}_{0.5}$ is the half-maximal activation potential and $\mathrm{k}$ is the slope factor.

Steady-state inactivation curves were generated using a Boltzmann equation.

$$
\frac{\mathrm{I}}{\mathrm{I}_{\max }}=\frac{1}{1+\exp \left(\mathrm{V}-\mathrm{V}_{0.5}\right) / \mathrm{k}}
$$

Where I is the current at indicated test pulse, $I_{\max }$ is the maximal current of Cav2.2 activation during test-pulse, $\mathrm{V}$ is the test potential, $\mathrm{V}_{0.5}$ is the half-maximal inactivation potential and $\mathrm{k}$ is the slope factor.

Recovery curves from close-state inactivation were the results from seven to nine independent experiments where series of recovery traces from inactivation time points were acquired. The data were fit using a single exponential of the following equation.

$$
\frac{\mathrm{I}}{\mathrm{I}_{\max }}=\left(\mathrm{y}_{0}-1\right) * \exp \left(-\frac{\mathrm{t}}{\tau}\right)+1
$$

Where $\mathrm{I}$ is the current at indicated time delay, $I_{\max }$ is the current at $512 \mathrm{~ms}$ of time delay, $\mathrm{y}_{0}$ is the non-inactivated current at $-40 \mathrm{mV}$ of pre-pulse, $\mathrm{t}$ is the time (the delay between prepulse and test-pulse) and $\mathrm{T}$ is the time constant of recovery from close-state inactivation. Statistical significance $(P<0.05)$ was determined using unpaired Student's t-tests or oneway ANOVA with Tukey's post hoc test.

\section{Expression and purification of human Cav2.2- $\alpha 2 \delta 1-\beta 1$ complex}

The DNA fragments of human Cav2.2 (UniProtKB accession: Q00975), $\alpha 2 \delta 1$ (UniProtKB accession: P54289) and $\beta 1$ (UniProtKB accession: Q02641) were amplified from a human cDNA library. The Cav2.2 (full-length wide-type or N-terminal 64-residue truncated), $\alpha 2 \delta 1$ and $\beta 1$ subunits were subcloned into a modified pEG BacMam vector. A superfolder GFP (sfGFP) and a Twin-Strep tag were tandemly attached at the C-terminus of the Cav2.2 for monitoring protein expression and affinity purification, respectively. The components of Cav2.2 complex were co-expressed in HEK 293F cells using the Bac-to-Bac baculovirus expression system. P1 and P2 viruses of each subunits were obtained using Sf9 insect cells. The P2 viruses were used to infect HEK 293F cells supplemented with $1 \%$ $(\mathrm{v} / \mathrm{v})$ fetal bovine serum when the cells density reached $2.5 \times 10^{6}$ cells $/ \mathrm{mL}$. The infected 
HEK 293F cells were cultured at $37^{\circ} \mathrm{C}$ in suspension and $5 \% \mathrm{CO}_{2}$ in a shaking incubator. 10 $\mathrm{mM}$ sodium butyrate was added after 12 hours. The cells were harvested after 48 hours and were stored at $-80^{\circ} \mathrm{C}$ immediately after being frozen in liquid nitrogen.

Cells expressing Cav2.2 complex were resuspended and broken in a purification buffer (20 mM HEPES pH 7.5, 150 mM NaCl, 5 mM $\beta$-mercaptoethanol ( $\beta$-ME), aprotinin (2 $\mu \mathrm{g} / \mathrm{mL})$, leupeptin $(1.4 \mu \mathrm{g} / \mathrm{mL})$, pepstatin $\mathrm{A}(0.5 \mu \mathrm{g} / \mathrm{mL}))$ using a Dounce homogenizer. Subsequently, the membranes were collected by centrifugation at $100,000 \times \mathrm{g}$ for $1 \mathrm{~h}$ and solubilized in the solubilization buffer (20 mM HEPES pH 7.5, $150 \mathrm{mM} \mathrm{NaCl}, 5 \mathrm{mM} \beta$ mercaptoethanol ( $\beta-M E), 1 \%(w / v)$ n-Dodecyl $\beta$-D-maltoside (DDM, Anatrace), 0.2\% (w/v) cholesteryl hemisuccinate (CHS, Anatrace)) at $4^{\circ} \mathrm{C}$ for $2 \mathrm{~h}$ with rotation. The insoluble cell debris was removed by centrifugation at $100,000 \times \mathrm{g}$ for $1 \mathrm{~h}$. The resulting supernatant was collected and passed through $2 \mathrm{~mL}$ Streptactin Beads, which was pre-equilibrated with the wash buffer (20 mM HEPES pH 7.5, 150 mM NaCl, $5 \mathrm{mM} \beta-\mathrm{ME}$ and 0.03\% (w/v) glycodiosgenin (GDN, Anatrace)) and washed with 10 column volumes of wash buffer supplemented with $2 \mathrm{mM}$ ATP and $5 \mathrm{mM} \mathrm{MgCl}_{2}$. The Cav2.2 complex was eluted with the wash buffer supplemented with $5 \mathrm{mM}$ desthiobiotin and $1 \mathrm{mM} \mathrm{CaCl}_{2}$, and then concentrated in the 100 kDa MW cut-off spin concentrators (Merck Millipore, Germany). For further purification, the concentrated protein sample was subjected to a Superose 6 Increase 10/300 GL gel filtration column (GE Healthcare, USA) pre-equilibrated in the wash buffer supplemented with $1 \mathrm{mM} \mathrm{CaCl}_{2}$. The peak fractions between $11.5 \mathrm{~mL}$ and $13.5 \mathrm{~mL}$ were pooled and concentrated to about $1.8 \mathrm{mg} / \mathrm{mL}$ for cryo-EM sample preparation.

\section{Cryo-EM sample preparation and data collection}

A droplet of $2.5 \mu \mathrm{L}$ of purified Cav2.2 complex was applied on the Quantifoil 1.2-1.3 Au 300 mesh grids glow-discharged for $60 \mathrm{~s}$ under $\mathrm{H}_{2}-\mathrm{O}_{2}$. The grids were then blotted for 4-5 s at $4{ }^{\circ} \mathrm{C}$ under condition of $100 \%$ humidity and vitrified in liquid ethane using a Vitrobot Mark IV.

Cryo-EM data were collected on a 300-kV microscope using a K2 Summit direct electron detector and a GIF Quantum LS energy filter. The slit was set to $20 \mathrm{eV}$. Movie stacks were acquired at a calibrated magnification of $105,000 \times$ in the super-resolution 
mode, with defocus values ranging from -1.2 to $-2.2 \mu \mathrm{m}$. The pixel size on motioncorrected micrographs was $1.04 \AA$. The micrographs were collected under a dose rate of $\sim 9.6 \mathrm{e}^{-} /\left(\AA^{2} \mathrm{~s}\right)$ and dose-fractioned in 32 frames, yielding a total accumulated dose of $\sim 60$ $\mathrm{e}^{-} / \AA^{2}$.

\section{Data Processing}

A total of 2,300 micrographs were motion-corrected and dose-weighted using MotionCor2 with $5 \times 5$ patches $^{39}$, followed by CTF estimation using GCTF ${ }^{40}$ and particle picking using both blob picker and template picker in cryoSPARC ${ }^{41}$. Several rounds of 2D and 3D classifications were performed in RELION to remove junk particles ${ }^{42}$. Initial multireference 3D classification generated 6 classes. The class 5 accounts for $26.1 \%$ of total particles, displaying discernible structural features, including the secondary structure elements of the $\alpha, \beta 1$ and $\alpha 2 \delta 1$ subunits. This class were selected and subjected to another round of $3 \mathrm{D}$ classification, giving rise to two classes with visible and continuous transmembrane helices. The particles from these two new classes were subjected to 3D refinement, generating a 3.5-Å resolution map. Subsequently, one more round of 3D classification was performed without further particle alignment. Two of the most populated classes were used for further 3D refinement, Bayesian polishing, and CTF refinement in RELION. The particles were then imported into cryoSPARC and subjected to Non-uniform Refinement. The final map was reported at $2.8-\AA \AA$ resolution according to golden standard Fourier shell correlation (GSFSC) criterion.

\section{Model building}

The cryo-EM map of Cav2.2 complex showed clear densities for most side chains and $\mathrm{N}$-glycans, which allowed us to reliably build and adjust the model. The homology models of $\alpha$ and $\alpha 2 \delta 1$ subunits were extracted from the structures of 0 . cuniculus Cav1.1 complex (PDB IDs: $5 G J^{43}$ and 7JPX ${ }^{44}$ ). The homology models of $\beta$ subunits were extracted from the structures of $R$. norvegicus $\mathrm{Cav}_{2} \beta_{2 \alpha}$ (PDB ID: 1T0J ${ }^{45}$ ). All of these homology models were fitted into the cryo-EM map as rigid bodies using the UCSF Chimera ${ }^{46}$. The resulting model was then manually inspected and adjusted in COOT ${ }^{47}$, followed by refinement 
against the cryo-EM map in real space using the phenix.real_space_refine utility. The model stereochemistry was evaluated using the Comprehensive validation (cryo-EM) utility in the PHENIX software package ${ }^{48}$.

All figures were prepared with ChimeraX or PyMOL (Schrödinger, LLC) ${ }^{49,50}$.

\section{Data availability}

The three-dimensional cryo-EM density maps of the Cav2.2 complex have been deposited in the Electron Microscopy Data Bank under the accession code EMD-xxxx. The coordinates for the Cav2.2 complex have been deposited in Protein Data Bank under accession code $x x x x$.

\section{Author contribution}

Y.Z. and D.J. conceived the project. Y.D. carried out molecular cloning and cell biology experiments. Y.D., Y.W., Y.L. and B.Y. expressed, purified protein complex sample and prepared sample for cryo-EM study. Y.D., Y.G. and Y.W. carried out cryo-EM data collection. Y.G. Z.Y.Y. and Y.Z. processed the cryo-EM data and prepared figures. Y.W. and B.L. built and refined the atomic model. Z.H. and Y.Z. designed and S.X. performed electrophysiological experiments. X.C.Z., D.J. and Y.Z. analyzed the structure. Y.Z. prepared initial draft of the manuscript. X.C.Z., D.J., Z.H. and Y.Z. edited the manuscript with input from all authors in the final version.

\section{Acknowledgments}

We thank X. Huang, B. Zhu, X. Li, L. Chen, and other staff members at the Center for Biological Imaging (CBI), Core Facilities for Protein Science at the Institute of Biophysics, Chinese Academy of Science (IBP, CAS) for the support in cryo-EM data collection. We thank Yan Wu for his research assistant service. This work is funded by Chinese Academy of Sciences Strategic Priority Research Program (Grant XDB37030304 to Y.Z. and Grant XDB37030301 to X.C.Z), the National Natural Science Foundation of China (31971134 to X.C.Z., 81371432 to Z.H.) and Institute of Physics, Chinese Academy of Sciences 


\section{(E0VK101 to D.J.).}

\section{Conflict of interest}

All authors declare that there is no conflict of interest that could be perceived as prejudicing the impartiality of the research reported.

\section{Reference}

1 Reuter, H. Properties of two inward membrane currents in the heart. Annual review of physiology 41 , 413-424 (1979).

2 Catterall, W. A. Excitation-contraction coupling in vertebrate skeletal muscle: a tale of two calcium channels. Cell 64, 871-874, doi:10.1016/0092-8674(91)90309-m (1991).

3 Tsien, R. W., Lipscombe, D., Madison, D. V., Bley, K. R. \& Fox, A. P. Multiple types of neuronal calcium channels and their selective modulation. Trends Neurosci 11, 431-438, doi:10.1016/01662236(88)90194-4 (1988).

Catterall, W. A. \& Few, A. P. Calcium channel regulation and presynaptic plasticity. Neuron 59, 882901, doi:10.1016/j.neuron.2008.09.005 (2008).

Nowycky, M. C., Fox, A. P. \& Tsien, R. W. Three types of neuronal calcium channel with different calcium agonist sensitivity. Nature 316, 440-443 (1985).

Snutch, T. P. \& Reiner, P. B. Ca2+ channels: diversity of form and function. Current opinion in neurobiology 2, 247-253 (1992).

7 Catterall, W. A., Perez-Reyes, E., Snutch, T. P. \& Striessnig, J. International Union of Pharmacology. XLVIII. Nomenclature and structure-function relationships of voltage-gated calcium channels. Pharmacological reviews 57, 411-425 (2005).

8 Olivera, B. M., Miljanich, G. P., Ramachandran, J. \& Adams, M. E. Calcium channel diversity and neurotransmitter release: the $\omega$-conotoxins and $\omega$-agatoxins. Annual review of biochemistry 63, 823867 (1994).

Westenbroek, R. E. et al. Biochemical properties and subcellular distribution of an N-type calcium channel alpha 1 subunit. Neuron 9, 1099-1115, doi:10.1016/0896-6273(92)90069-p (1992).

Westenbroek, R. E., Hoskins, L. \& Catterall, W. A. Localization of Ca2+ channel subtypes on rat spinal motor neurons, interneurons, and nerve terminals. J Neurosci 18, 6319-6330, doi:10.1523/jneurosci.18-16-06319.1998 (1998).

11 Weiss, N. The first disease connection for Cav2.2 channels. Gen Physiol Biophys 34, 217-219, doi:10.4149/gpb_2015014 (2015). $\mathrm{Wu}$, J. et al. Structure of the voltage-gated calcium channel Ca v 1.1 at 3.6 Å resolution. Nature 537, 191-196 (2016).

Zhao, Y. et al. Molecular basis for ligand modulation of a mammalian voltage-gated Ca2+ channel. Cell 177, 1495-1506. e1412 (2019).

Gao, S. \& Yan, N. Structural Basis of the Modulation of the Voltage-Gated Calcium lon Channel Cav1. 1 by Dihydropyridine Compounds. Angewandte Chemie 133, 3168-3174 (2021).

Zhao, Y. et al. Cryo-EM structures of apo and antagonist-bound human Ca v 3.1. Nature 576, 492-497 
(2019).

17 Patil, P. G., Brody, D. L. \& Yue, D. T. Preferential closed-state inactivation of neuronal calcium channels. Neuron 20, 1027-1038 (1998).

18 Jones, L. P., DeMaria, C. D. \& Yue, D. T. N-type calcium channel inactivation probed by gating-current analysis. Biophysical journal 76, 2530-2552 (1999).

19 Thaler, C., Gray, A. C. \& Lipscombe, D. Cumulative inactivation of N-type CaV2. 2 calcium channels modified by alternative splicing. Proceedings of the National Academy of Sciences 101, 5675-5679 (2004).

20 Suha, B.-C., Kima, D.-I., Falkenburgerb, B. H. \& Hilleb, B. Membrane-localized $\beta$-subunits alter the PIP2 regulation of high-voltage activated Ca 2 channels. PNAS 109, 3161-3166 (2012).

21 Vivas, O., Castro, H., Arenas, I., Elías-Viñas, D. \& García, D. E. PIP2 hydrolysis is responsible for voltage independent inhibition of CaV2. 2 channels in sympathetic neurons. Biochemical and biophysical research communications 432, 275-280 (2013). channels by Gq-coupled receptor is facilitated by membrane-localized $\beta$ subunit. $J$ Gen Physiol 144, 297-309, doi:10.1085/jgp.201411245 (2014). Hille, B., Dickson, E. J., Kruse, M., Vivas, O. \& Suh, B.-C. Phosphoinositides regulate ion channels. Biochimica et biophysica acta 1851, 844-856, doi:10.1016/j.bbalip.2014.09.010 (2015).

24 Wu, L., Bauer, C. S., Zhen, X.-g., Xie, C. \& Yang, J. Dual regulation of voltage-gated calcium channels by Ptdlns(4,5)P2. Nature 419, 947-952, doi:10.1038/nature01118 (2002). Ion Channels by PIP2. Frontiers in Pharmacology 3, doi:10.3389/fphar.2012.00170 (2012). Chanda, B. \& Bezanilla, F. Tracking voltage-dependent conformational changes in skeletal muscle sodium channel during activation. J Gen Physiol 120, 629-645, doi:10.1085/jgp.20028679 (2002). Xia, F. et al. Inhibition of cholesterol biosynthesis impairs insulin secretion and voltage-gated calcium channel function in pancreatic beta-cells. Endocrinology 149, 5136-5145, doi:10.1210/en.2008-0161 (2008). channels and BK-type potassium channels in auditory hair cells. PloS one 6, e26289-e26289, doi:10.1371/journal.pone.0026289 (2011).

Guo, J. et al. Structure of the voltage-gated two-pore channel TPC1 from Arabidopsis thaliana. Nature 531, 196-201 (2016).

30 Kintzer, A. F. \& Stroud, R. M. Structure, inhibition and regulation of two-pore channel TPC1 from Arabidopsis thaliana. Nature 531, 258-264 (2016).

31 Wisedchaisri, G. et al. Resting-State Structure and Gating Mechanism of a Voltage-Gated Sodium Channel. Cell 178, 993-1003.e1012, doi:10.1016/j.cell.2019.06.031 (2019). Zhang, X. C., Yang, H., Liu, Z. \& Sun, F. Thermodynamics of voltage-gated ion channels. Biophysics Reports 4, 300-319, doi:10.1007/s41048-018-0074-y (2018).

Rodriguez-Menchaca, A. A. et al. PIP\&lt;sub\&gt;2\&lt;/sub\&gt; controls voltage-sensor movement and pore opening of Kv channels through the S4-S5 linker. Proceedings of the National Academy of Sciences 109, E2399, doi:10.1073/pnas.1207901109 (2012). regulation of high-voltage activated Ca2+ channels. Proc Natl Acad Sci U S A 109, 3161-3166, doi:10.1073/pnas.1121434109 (2012). 

variants lacking the synaptic protein interaction site. Journal of Neuroscience 22, 82-92 (2002). Catterall, W. A., Leal, K. \& Nanou, E. Calcium channels and short-term synaptic plasticity. The Journal of biological chemistry 288, 10742-10749, doi:10.1074/jbc.R112.411645 (2013).

37 McArthur, J. R. et al. Inhibition of human N- and T-type calcium channels by an ortho-phenoxyanilide derivative, MONIRO-1. Br J Pharmacol 175, 2284-2295, doi:10.1111/bph.13910 (2018). Liu, Y. et al. CDYL suppresses epileptogenesis in mice through repression of axonal Nav1.6 sodium channel expression. Nature communications 8, 355, doi:10.1038/s41467-017-00368-z (2017). Zheng, S. Q. et al. MotionCor2: anisotropic correction of beam-induced motion for improved cryoelectron microscopy. Nature methods 14, 331-332 (2017).

40 Zhang, K. Gctf: Real-time CTF determination and correction. Journal of structural biology 193, 1-12 (2016).

41 Punjani, A., Rubinstein, J. L., Fleet, D. J. \& Brubaker, M. A. cryoSPARC: algorithms for rapid unsupervised cryo-EM structure determination. Nature methods 14, 290-296 (2017). Zivanov, J. et al. New tools for automated high-resolution cryo-EM structure determination in RELION3. elife 7, e42166 (2018). $\mathrm{Wu}, \mathrm{J}$. et al. Structure of the voltage-gated calcium channel $\mathrm{Ca}(\mathrm{v}) 1.1$ at $3.6 \AA$ resolution. Nature 537, 191-196, doi:10.1038/nature19321 (2016).

44 Madej, T. et al. MMDB and VAST+: tracking structural similarities between macromolecular complexes. Nucleic acids research 42, D297-303, doi:10.1093/nar/gkt1208 (2014). Van Petegem, F., Clark, K. A., Chatelain, F. C. \& Minor, D. L., Jr. Structure of a complex between a voltage-gated calcium channel beta-subunit and an alpha-subunit domain. Nature 429, 671-675, doi:10.1038/nature02588 (2004).

Pettersen, E. F. et al. UCSF Chimera-a visualization system for exploratory research and analysis. Journal of computational chemistry 25, 1605-1612 (2004).

47 Emsley, P. \& Cowtan, K. Coot: model-building tools for molecular graphics. Acta crystallographica section D: biological crystallography 60, 2126-2132 (2004).

48 Adams, P. D. et al. PHENIX: a comprehensive Python-based system for macromolecular structure solution. Acta Crystallographica Section D: Biological Crystallography 66, 213-221 (2010).

49 Goddard, T. D. et al. UCSF ChimeraX: Meeting modern challenges in visualization and analysis. Protein Science 27, 14-25 (2018).

50 DeLano, W. L. Pymol: An open-source molecular graphics tool. CCP4 Newsletter on protein crystallography 40, 82-92 (2002). 
a

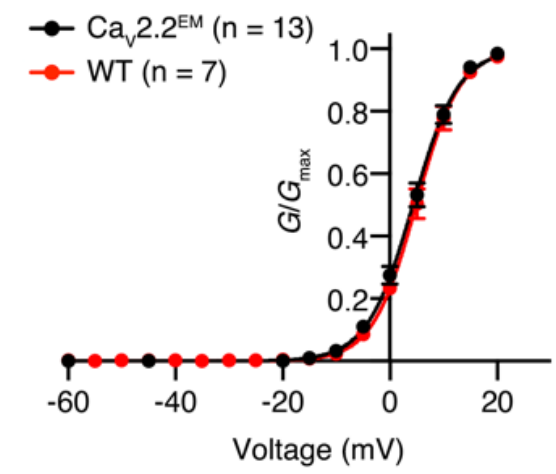

C

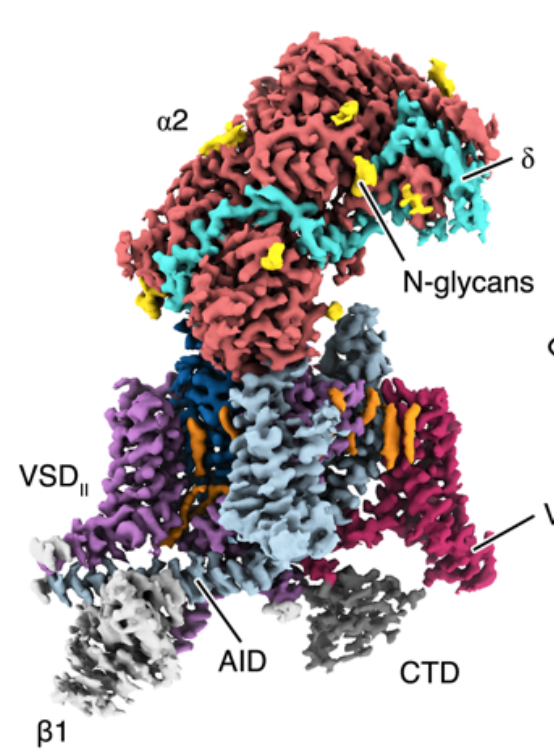

b

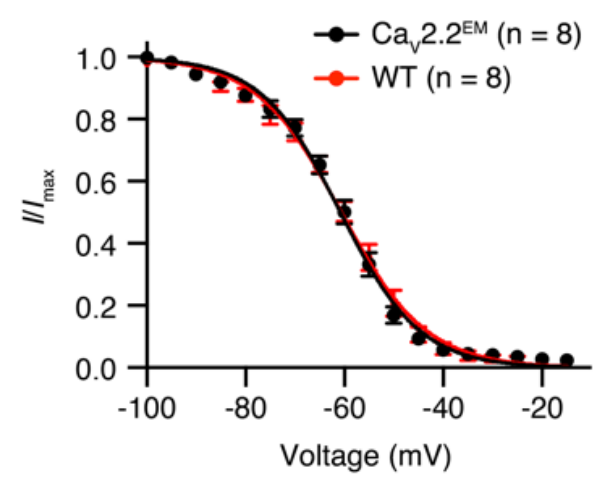

d

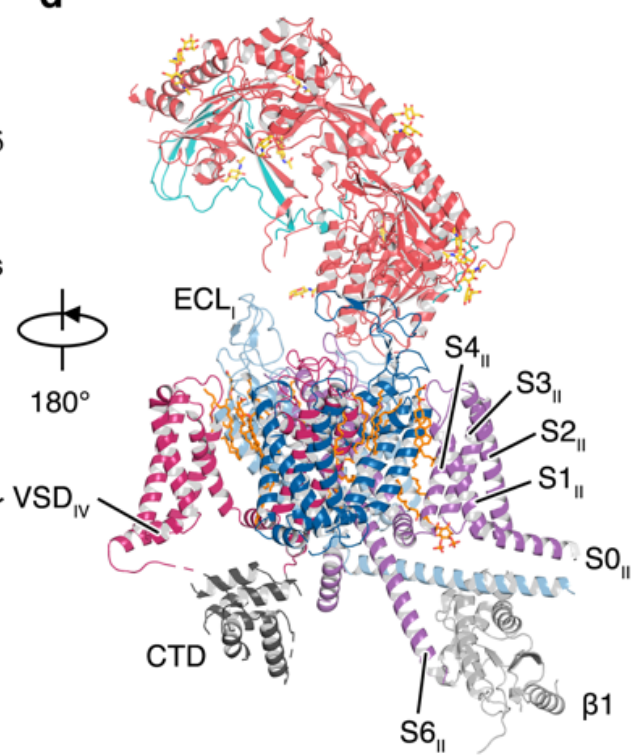

2 Figure 1. Architecture of the Cav2.2 complex.

3 a. Normalized conductance-voltage (G/V) relationship and the Boltzmann fits for the N-terminal 4 truncated Cav2.2 ${ }^{\mathrm{EM}}$ complex and full-length wild type (WT) Cav2.2 complex. HEK 293T cells 5 expressing the complex were stimulated with 200-ms depolarizing pulses between $-60 \mathrm{mV}$ and 50 $6 \mathrm{mV}$ in steps of $10 \mathrm{mV}$ from a holding potential of $-100 \mathrm{mV}$. b. Steady-state inactivation of the WT 7 Cav2.2 complex and the Cav2.2 ${ }^{\mathrm{EM}}$ complex. Cells were stepped from a holding potential of $-100 \mathrm{mV}$ 8 to pre-pulse potentials between $-100 \mathrm{mV}$ and $-15 \mathrm{mV}$ in $5 \mathrm{mV}$ increments for $10 \mathrm{~s}$. Black, Cav2.2 ${ }^{\mathrm{EM}}$ 9 complex; red, full-length wild-type Cav2.2 complex. c-d. The density map and model of the Cav2.2 10 complex as seen in parallel to the membrane plane. The $\alpha 2 \delta 1$ and $\beta 1$ subunit, C-terminal domain 11 (CTD), extracellular loops (ECL), alpha-interacting domain (AID), and transmembrane helices S1 II$12 S 4_{\|}$in $V S D_{\|}$were labeled. The $\alpha 1$ subunit is colored in light blue $\left(D_{1}\right)$, violet $\left(D_{\|}\right)$, deep blue $\left(D_{\| I}\right)$, 13 magenta ( $\left.D_{\mathrm{IV}}\right)$, and grey (CTD) respectively. The $\beta 1, \alpha 2$ and $\delta 1$ subunits are colored in light-grey, 14 tomato-red, and turquoise, respectively. $\mathrm{N}$-glycans is displayed and colored in gold. 
a

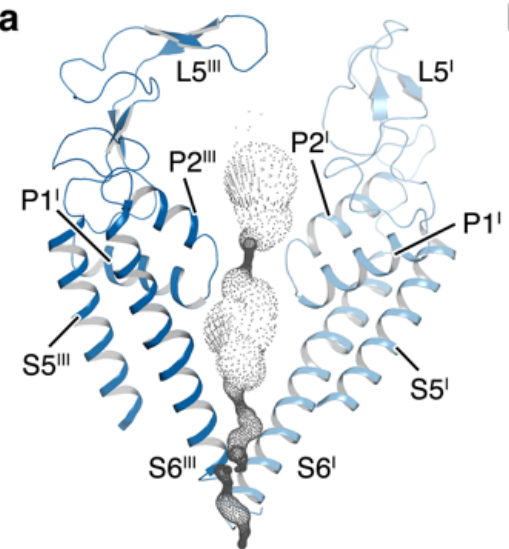

d

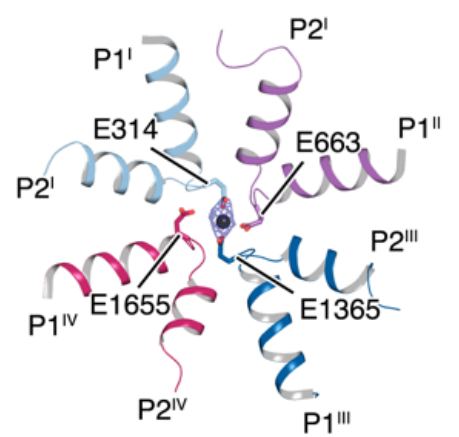

b

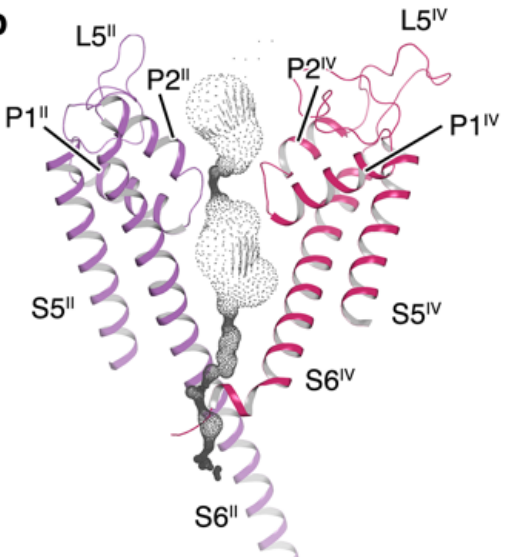

e
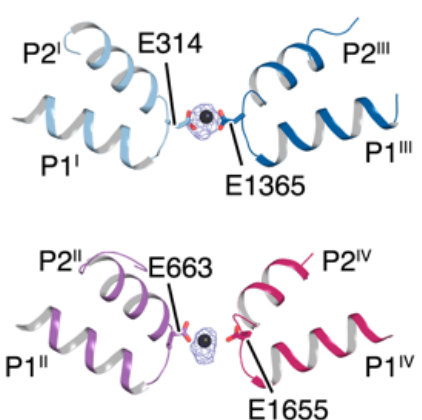

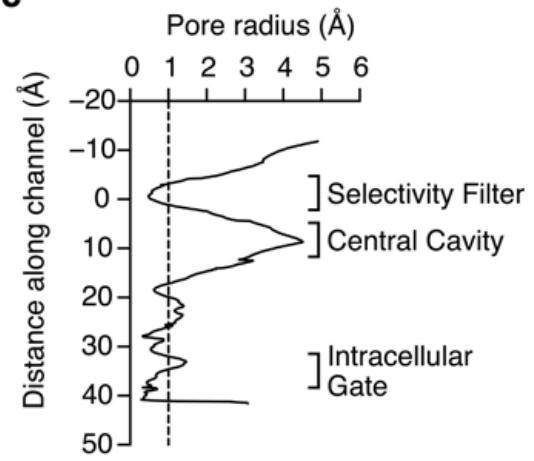

f

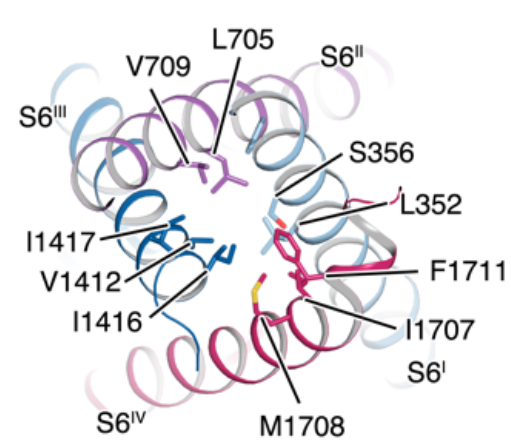

Figure 2. Ion conduction pore of the Cav2.2

18 a-b. Ion permeation pathways calculated by the program HOLE were shown in dots. The selectivity

19 filter and S5-S6 helices are shown in cartoon and viewed in parallel to the membrane plane. c. Plot 20 of pore radii for Cav2.2 complex. Vertical dashed line marks $1.0 \AA$ pore radius. $\mathbf{d}-\mathbf{e}$. "Top-down" and 21 side-view of the selectivity filter. The selectivity filter ring of four glutamate residues from the four 22 domains of $\mathrm{Ca}_{v}$ channel were shown in sticks. A cation ion is shown as a grey sphere, overlaid with 23 corresponding EM density colored in marine. f. The intracellular gate formed by four S6 helix viewed 24 from intracellular side. Hydrophobic residues are shown in sticks. The segments from $D_{\text {I, }} D_{\text {II, }} D_{\text {III }}$ and $25 D_{\mathrm{IV}}$ in the Cav2.2 complex are colored in light blue, violet, deep blue, and magenta, respectively. 


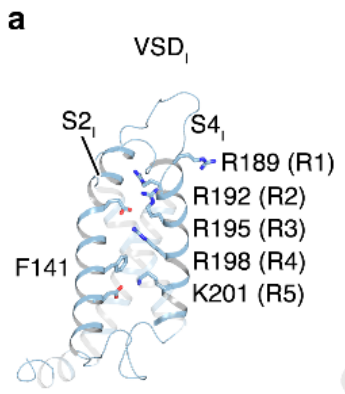

So, b

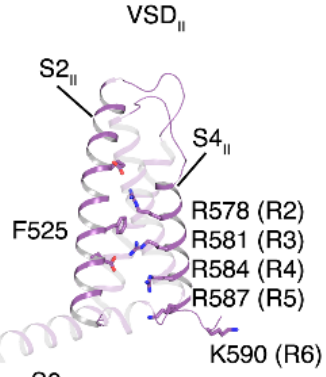

$\mathrm{SO}_{\|}$

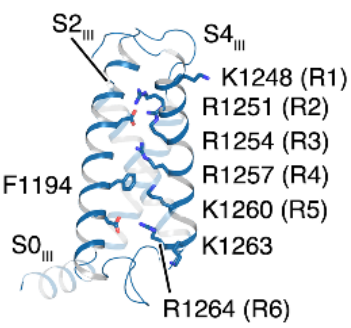

d

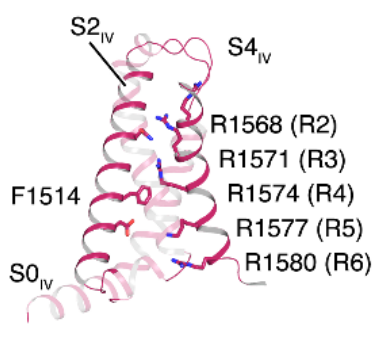

e

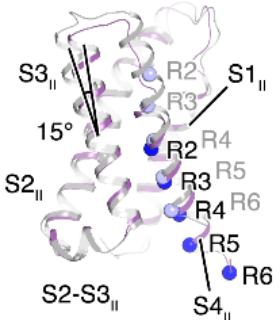

f

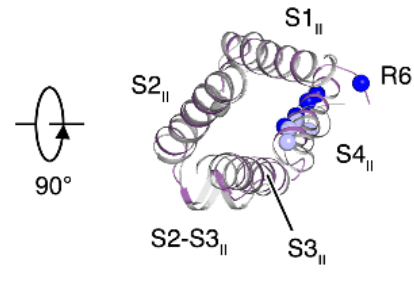

g

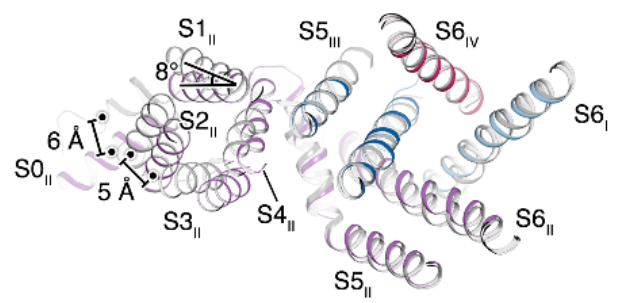

h

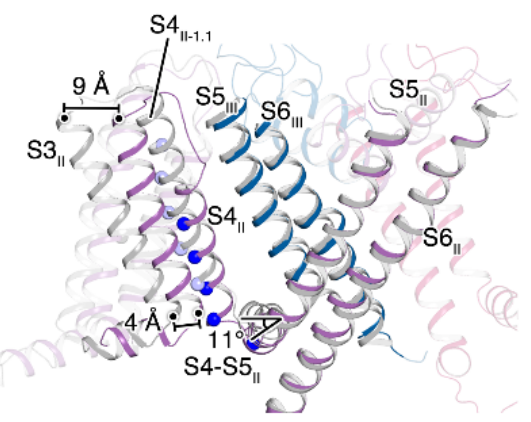

i

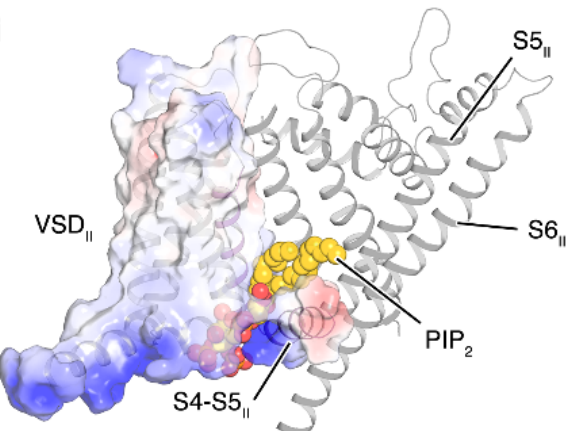

28 Figure 3. Structural analysis of the voltage-sensing domains.

29 a-d. Voltage sensing domains from $D_{I}(\mathbf{a}), D_{I I}(\mathbf{b}), D_{I I I}(\mathbf{c})$ and $D_{I V}(\mathbf{d})$ are shown in cartoon. The gating30 charge residues (R1-R6) on S4 helix and residues from surrounding helices are shown in sticks. e-f.

31 Structural comparison of the $\mathrm{VSD}_{\|}$at resting state from Cav2.2 complex and activated state from 32 Cav1.1 complex, viewed parallel (e) or perpendicular (f) to the membrane plane. The gating-charge 33 residues are shown as spheres, colored in deep blue (Cav2.2) or light blue (Cav1.1). $\mathbf{g}-\mathbf{h}$. 34 superimposition of the $\mathrm{VSD}_{\|}{ }^{\mathrm{R}}$ and $\mathrm{VSD}_{\|}{ }^{\mathrm{A}}$ using $\mathrm{S} 5-\mathrm{S} 6$ helices as a reference, viewed perpendicular 35 (g) or parallel $(\mathbf{h})$ to the membrane plane. i. Domain II of the Cav2.2 complex, overlaid with an 36 electrostatic potential surface on the $\mathrm{VSD}_{\|}$. The putative $\mathrm{PIP}_{2}$ molecular is shown as spheres. 
a

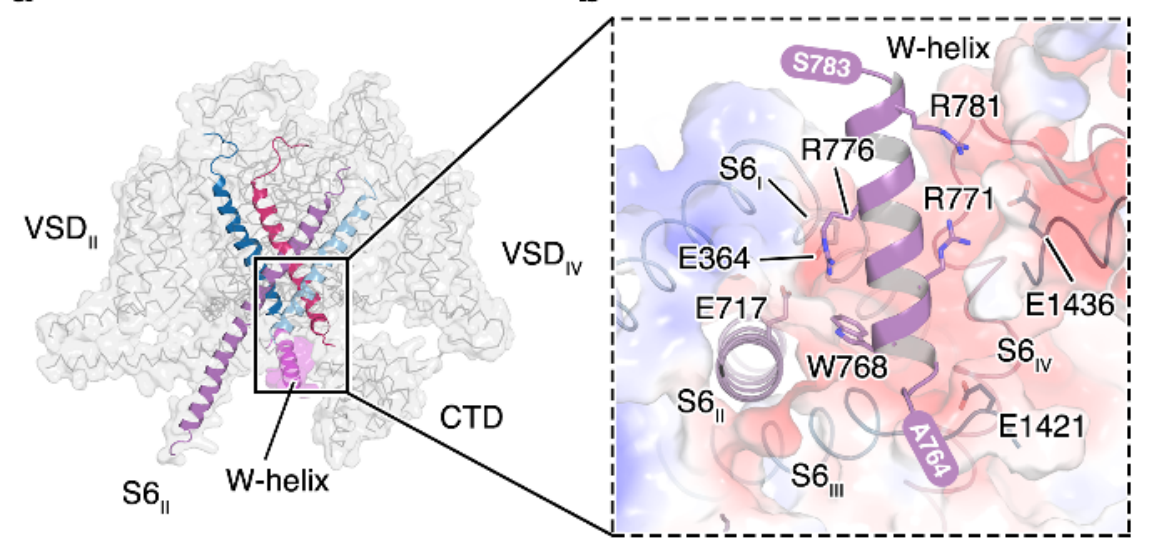

d

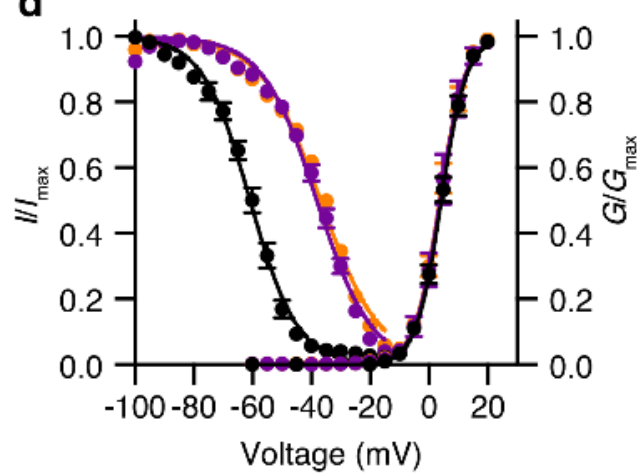

e

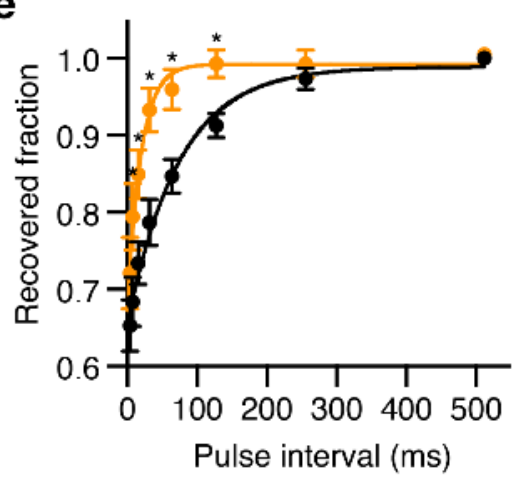

C

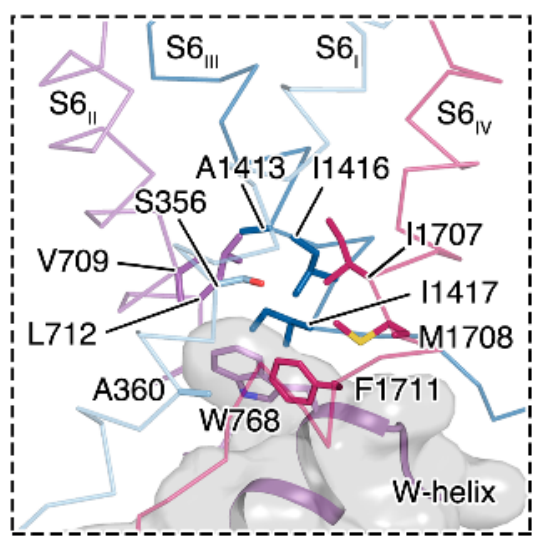

f

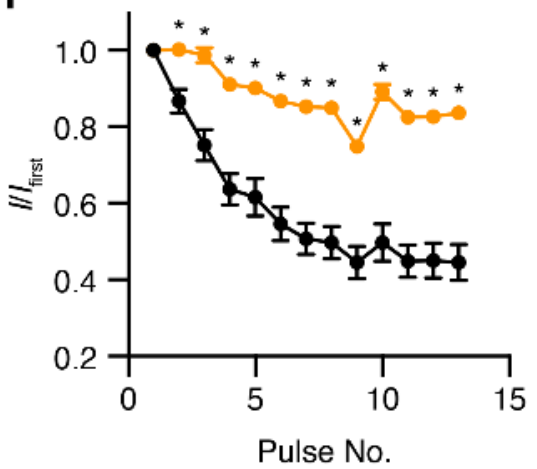

38

Figure 4. Inactivation mechanism of Cav2.2 complex.

$\rightarrow \Delta$ W-Helix $\rightarrow$ W768Q

a. The W-helix is composed of ${ }^{764} A R S V W E Q R A S Q L R L Q N L R A S^{783}$ and determined underneath the intracellular gate of the Cav2.2. The Cav2.2 is shown in ribbon and overlaid with transparent surfaces. The $\mathbf{S 6}$ helices are shown in cartoon and highlighted. The W-helix is shown in cartoon and overlaid with a transparent surface, colored in purple. $\mathbf{b}$. Interactions between the W-helix and the intracellular gate viewed from intracellular side. The critical residues involved in the interactions were shown in sticks. The $\mathrm{N}$ - and C-terminus of the W-helix is indicated. c. The W768 from W-helix stabilized the intracellular gate at closed state. The $\mathrm{S} 6$ helices are shown as ribbon. The W-helix is shown in cartoon and overlaid with a transparent grey surface. d. Steady-state activation and inactivation for the Cav2. ${ }^{\mathrm{EM}}$ complex and its mutants. (Activation: $\mathrm{EM}, \mathrm{n}=13 ; \Delta \mathrm{W}$-helix, $\mathrm{n}=8$; W768Q, $n=6$; Inactivation: $E M, n=8 ; \Delta W$-helix, $n=6$; W768Q, $n=6$ ) e. Recovery of close-state inactivation for the Cav2.2 ${ }^{\mathrm{EM}}$ complex and its mutants. Cells were held to $-40 \mathrm{mV}$ for $200 \mathrm{~ms}$, and then stepped to $-100 \mathrm{mV}$ for indicated time delay (4-512 ms), followed by a $+10 \mathrm{mV}$ test-pulse $(35 \mathrm{~ms})(E M, n=7 ; \Delta W$-helix, $n=$ 8). f. Ratio of Cav2.2 channels inactivation calculated from the first spike eliciting maximal current to the other spikes in the AP trains ( $E M, n=8 ; \Delta W$-helix, $n=6$ ). Black, Cav2.2 ${ }^{\mathrm{EM}}$ complex; purple, W768Q; orange, $\Delta$ W-helix. ${ }^{*}$ indicates $p<0.05$. 